\title{
Peripheral facial nerve palsy in children in a Borrelia high endemic area: epidemiology and evaluation of clinical recovery. A retrospective one-year follow up.
}

Sigurdur Arnason MD'1, Malou Hultcrantz MD, PhD², Anna Nilsson MD, PhD ${ }^{3,4}$, Åsa Laestadius MD, PhD ${ }^{1,4}$

1. Astrid Lindgren Children's Hospital, Karolinska University Hospital, Stockholm, Sweden.

2. Division of Ear, Nose and Throat Diseases, Karolinska University Hospital, Stockholm, Sweden.

3. Childhood Cancer Research Unit, Q6:05, Dept of Women and Children's Health, Karolinska Institutet, Stockholm, Sweden.

4. Department of Women and Children's Health, Karolinska Institute, Stockholm, Sweden.

Introduction: Acute peripheral facial nerve palsy (FNP) in children is more common in Borrelia endemic areas. The incidence is 20-60/100.000/year. Children recover fully in $80-100 \%$ of cases.

Neuroborreliosis is the most common identifiable cause in children and significantly more common than in adults. Another cause is Bell's palsy (BP), however less common than in adults.

Objective: To identify the incidence, etiology and prognosis of FNP in children in the Borrelia high endemic region of Stockholm, Sweden.

Method: A retrospective study, identifying children from 1-18 years of age who visited the Pediatric Emergency Department with a history of FNP during a one-year period 2014 2015. Epidemiological data was collected from medical chart reviews where two different facial grading systems, the House-Brackmann (HB) and Sunnybrook (SB), were used to measure clinical outcome.

Results: We identified 77 children with FNP; an estimated incidence of $30-40 / 100000$ children/year. Forty-five children were diagnosed with neuroborreliosis $(58,4 \%), 28$ with BP $(36,4 \%)$ and four with other causes of FNP $(5,2 \%)$. Neuroborreliosis was almost only seen in children below 10 years of age and from June till November whereas BP had a more even seasonal and age distribution. Six patients $(7,8 \%)$ had remaining symptoms at least 3 months after onset. Four were 10 years or older (67\%), all had a SB score $\geq 80 / 100$ and/or a HB score $\leq 3$ upon their last registered visit. Three had BP (10-17y of age) one had neuroborreliosis (10y) and two had other causes of FNP.
Conclusion: Neuroborreliosis was the major cause of FNP during our study period and dominated in the younger ages. Children heal well from FNP but our data indicates that older children and children with BP may have a risk of slower and incomplete recovery as opposed to younger children with neuroborreliosis. The Sunnybrook grading scale may have a predictive value in children with FNP.

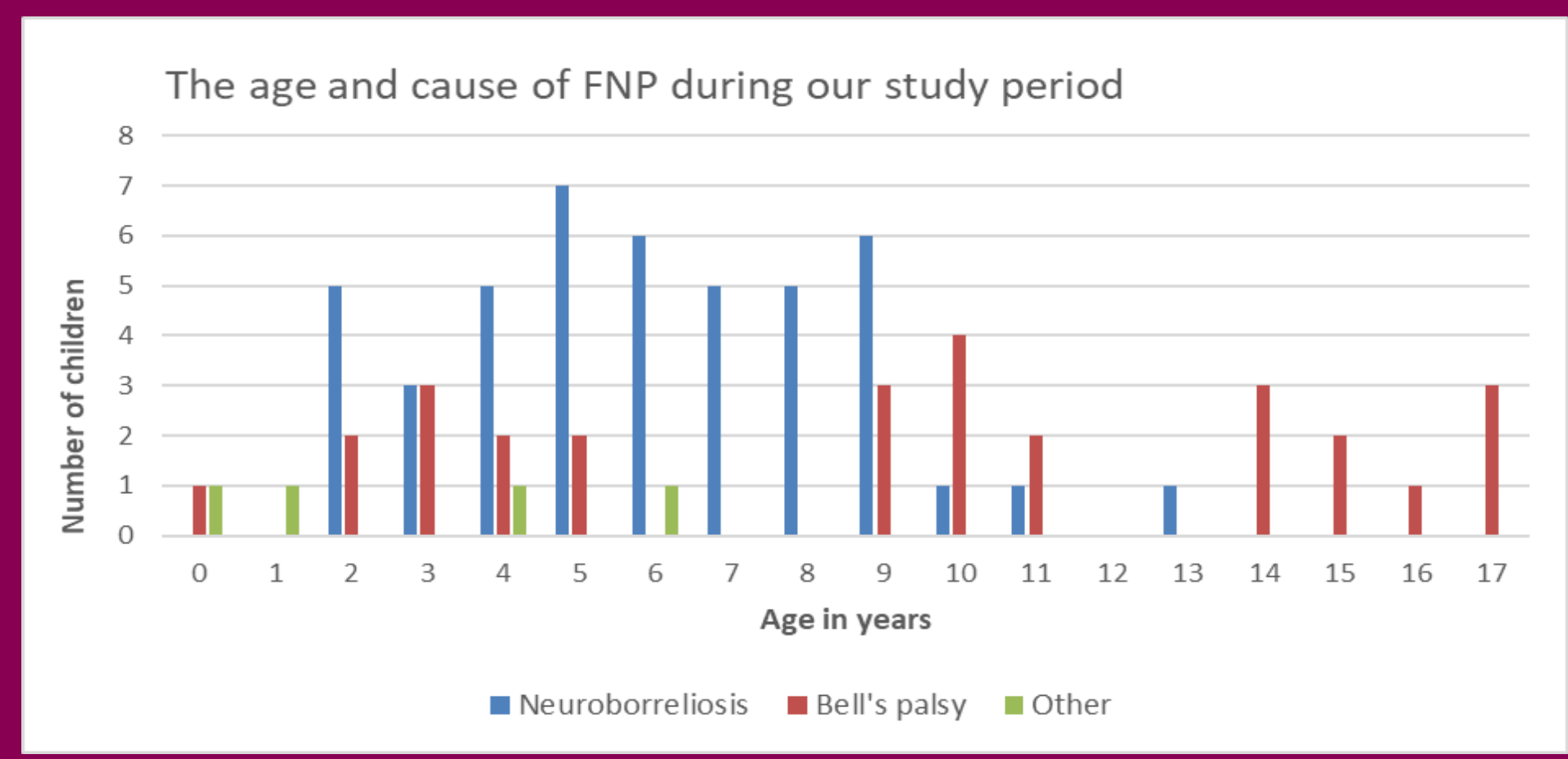

\begin{tabular}{|c|c|c|c|c|}
\hline Characteristic & Neuroborreliosis & Bell's palsy & Other & Total \\
\hline Diagnosis (all children) & $45(58,4 \%)$ & $29(36,4 \%)$ & $4(5,2 \%)$ & 77 (100\%) \\
\hline Median age & $6,5 y$ & $9,4 y$ & $3,1 y$ & $6,9 y$ \\
\hline Tick bite less than $6 \mathrm{w}$ ago & 7 & 0 & 0 & 7 \\
\hline Erythema Migrans (EM) & $\begin{array}{c}12(27 \% \text { of } \\
\text { Neuroborreliosis) }\end{array}$ & 0 & 0 & 12 \\
\hline Head and neck EM & 10 & 0 & 0 & 10/12 (83\%) \\
\hline Thorax EM & 1 & 0 & 0 & $1 / 12(8,5 \%)$ \\
\hline Unknown location of EM & 1 & 0 & 0 & $1 / 12(8,5 \%)$ \\
\hline
\end{tabular}

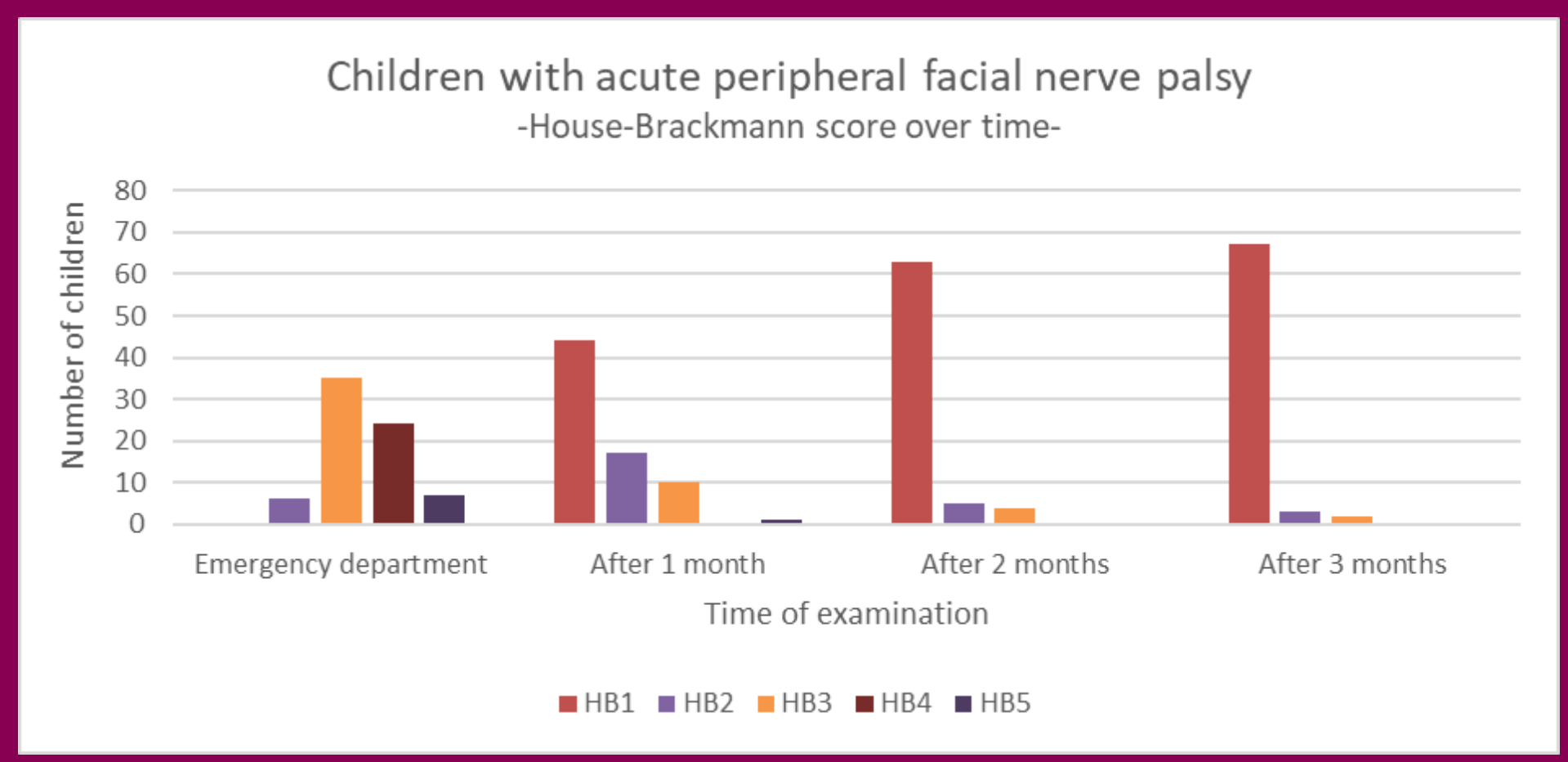

